\title{
Introducing New Guidelines on Sudden Unexpected Death in Epilepsy
}

\author{
An Expert Interview with Cynthia L Harden \\ Department of Neurology, Mount Sinai Health System, New York, NY, US
}

DOl: https://doi.org/10.17925/USN.2017.13.02.65

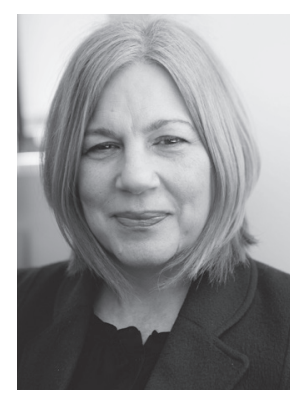

\section{Cynthia L Harden}

Cynthia L Harden, MD, received her medical degree at the University of Wisconsin. She trained in internal medicine at Mount sinai

St Luke's Hospital and neurology at Mount Sinai Hospital, both in New York City, and in clinical neurophysiology at Albert Einstein College of Medicine in the Bronx. She served most of her career at Weill Cornell College of Medicine, where she became Professor of Neurology. Dr Harden serves as Chair of the Guideline Development, Dissemination and Implementation Subcommittee of the American Academy of Neurology (AAN). In 2016, she was also elected Chair of AAN's Epilepsy Section for a 2-year term.

\section{Keywords}

Epilepsy, guidelines, sudden unexpected death, SUDEP

Disclosure: Cynthia L Harden has nothing to declare in relation to this article. This is an expert interview and as such, has not undergone the journal's standard peer review process. No funding was received in the publication of this article.

Authorship: All named authors meet the International Committee of Medical Journal Editors (ICMJE) criteria for authorship of this manuscript, take responsibility for the integrity of the work as a whole, and have given final approval for the version to be published.

open Access: This article is published under the Creative commons Attribution Noncommercial License, which permits any noncommercial use, distribution, adaptation, and reproduction provided the original author(s) and source are given appropriate credit.

Received: June 12, 2017

Published Online: July 24, 2017

Citation: US Neurology, 2017;13(2):65-6

Corresponding Author: Cynthia L Harden, Mount Sinai Health System, Mount Sinai Beth Israel Phillips Ambulatory Care Center, 10 Union Square East, Suite 5D, New York, NY 10003, US. E: Cynthia.Harden@mountsinai.org
$\mathrm{N}$ ew guidelines from the American Academy of Neurology (AAN) and the American Epilepsy Society on sudden unexpected death in epilepsy (SUDEP) were presented at the 69th Annual AAN Meeting held in Boston, MA, US, May 28-22, 2017 in parallel with their publication in Neurology. ${ }^{1}$ In an expert interview, Dr Cynthia L Harden of Mount Sinai discusses SUDEP, why the new guidelines were needed and the key recommendations.

\section{Q. What is the incidence of SUDEP?}

Based on moderate confidence in the evidence from three Class I studies, SUDEP risk in children with epilepsy is $0.22 / 1,000$ patient-years ( $95 \%$ confidence interval [Cl] 0.16-0.31). ${ }^{1}$ Based on low confidence in the evidence, due to considerable imprecision across five Class I studies, SUDEP risk increases in adults to $1.2 / 1,000$ patient-years $(95 \% \mathrm{Cl} 0.64-2.32){ }^{1}$

These rates were derived according to the AAN guideline process, as follows: in the comprehensive analysis of the literature, the authors included reports that met AAN criteria to be categorized as Class I, which means they are less likely to have selection bias than reports determined to be Class II-IV. ${ }^{2}$ The Class I articles surveyed a population-representative group of people with epilepsy, and captured at least $80 \%$ of the deaths in the study population, and of the deaths, at least $80 \%$ were systematically evaluated for death due to SUDEP. A random-effects meta-analysis was performed across age groups, due to variation in SUDEP incidence rates across studies. The three papers in children had close agreement as to the SUDEP incidence per 1,000 epilepsy patient-years, therefore the confidence in the evidence is "moderate", meaning that future studies are unlikely to change this incidence rate. The five papers in adults with epilepsy had more variation in their findings, resulting in the confidence in the evidence as "low", meaning that future studies may change this SUDEP incidence rate. These rates are based on an accurate documentation of the SUDEP diagnosis, so that the SUDEP occurrences were either "definite", meaning an autopsy was performed, or "probable", meaning that the SUDEP criteria were met, but no autopsy was performed. Overall, the incidence appears to increase after age 18. Patients over age 55 were often excluded in the reports since competing causes of death e.g., cardiovascular-related, could not be reliably excluded in this mature age group. These results suggest that ongoing research to accurately capture SUDEP diagnoses at the point of contact with the medical examiner, is still needed. 


\section{Q. What are known risk factors for SUDEP?}

The strongest risk factor for SUDEP is the occurrence of generalized tonicclonic seizures (GTCS). ${ }^{1}$ The risk increases in a 'dose-related' manner; the more frequently GTCS occur, the higher the risk of SUDEP. Compared with not having GTCS as part of the seizure type for a person with epilepsy, the risk of SUDEP increased five-fold if 1-2 GTCS occur per year, and 18-fold if more than three GTCS occur per year. Further, not trying a new treatment to reduce GTCS occurrence compared to actively trying a new therapeutic intervention was associated with a six-fold increased risk of SUDEP per year. On the side of reducing the risk of SUDEP, nocturnal supervision and using a nocturnal listening device decreased the risk of SUDEP by $60-90 \%$. These were the strongest modifiable risk factors for increasing or reducing the risk of SUDEP. No specific anti-seizure medication was associated with increasing the risk of SUDEP. The occurrence of nocturnal seizures and male gender both had low strength of supporting evidence for increasing the risk of SUDEP.

\section{Q. Why was a new guideline needed?}

The AAN was asked to undertake this guideline upon request from the organization called 'Partners Against Mortality in Epilepsy' which is now part of the American Epilepsy Society and includes patient advocacy representatives, physicians and nurses who care for patients with epilepsy, clinician-researchers, and basic science researchers. The body of knowledge regarding SUDEP and the need for awareness and discussion about SUDEP reached a critical mass on several fronts, prompting the next step in SUDEP academia, which was an evidence-based guideline on incidence and risk factors. The patient and family consumer groups have stated that they have a 'right to know' about SUDEP. The medical community has appropriately responded to this fundamental demand and developed this guideline. The AAN guideline process has also undergone major evolution since 2011, and has adopted the comprehensive Institute of Medicine approach. ${ }^{3}$ The conclusions now synthesize the strengths and drawbacks of the available evidence, and the recommendations derived from those conclusions are actionable, making them much more useful and applicable to the practice of neurology.

\section{Q. What does the new AAN/American Epilepsy Society guideline recommend in terms of SUDEP?}

There are five recommendations stated in the guideline. ${ }^{1}$
They are as follows:

- Clinicians caring for young children with epilepsy should inform parents and guardians that there is a rare risk of SUDEP. In 1 year, SUDEP typically affects one in 4,500 children with epilepsy.

- Clinicians should inform adult patients with epilepsy that there is a small risk of SUDEP. In 1 year, SUDEP typically affects one in 1,000 adults with epilepsy.

- For persons with epilepsy who continue to experience GTCS, clinicians should continue to actively manage epilepsy therapies to reduce seizures and SUDEP risk while incorporating patient preferences and weighing the risks and benefits of any new approach.

- Clinicians should inform persons with epilepsy that seizure freedom, particularly freedom from GTCS (which is more likely to occur with medication adherence), is strongly associated with a decreased SUDEP risk.

- For persons with frequent GTCS and nocturnal seizures, clinicians may advise selected patients and families, if permitted by their individualized epilepsy and psychosocial circumstances, to use nocturnal supervision or other nocturnal precautions, such as the use of a remote listening device, to reduce SUDEP risk.

\section{Q. What other factors may affect the risk of SUDEP?}

The strongest evidence supports the risk factors as outlined above. Other modifiable risk factors, supported by less strong evidence, include never having taken anti-seizure medication, and changing anti-seizure medication multiple times in a year. The latter may be a surrogate marker for difficultto-treat epilepsy, or for patients with poor tolerability to medications. However, the number of anti-seizure medications taken did not affect the risk of SUDEP, only the frequency of GTCS. The increased SUDEP risk imparted by GTCS occurring in the prone position, or by the duration of postictal generalized electroencephalogram suppression, is only weakly supported by the available evidence. Therefore, these factors did not lead to actionable recommendations. The guideline points out areas where more evidence is needed to support interventions to prevent SUDEP. While not providing an actual script for the practitioner to talk about SUDEP with epilepsy patients and their families, clear rates of occurrence of SUDEP, and some language to be used for this discussion, are included. Several important management strategies are also recommended, which are well supported by the available evidence regarding SUDEP risk. ${ }^{1}$

1. Harden C, Tomson T, Gloss D, et al., Practice guideline summary: sudden unexpected death in epilepsy incidence rates and risk factors: report of the Guideline Development, Dissemination, and Implementation Subcommittee of the American Academy of Neurology and the American Epilepsy Society, Neurology, 2017;88:1674-80.

2. AAN (American Academy of Neurology). 2011. Clinical Practice Guideline Process Manual, 2011 Ed. St. Paul, MN: The American Academy of Neurology. Available at: http://tools.aan.com/globals/axon/assets/9023.pdf (accessed July 2017).

3. IOM (Institute of Medicine). 2011. Finding What Works in Health Care: Standards for Systematic Reviews. Washington, DC: The National Academies Press. Available at: www.ncbi.nlm.nih.gov/books/NBK209518/pdf/ Bookshelf_NBK209518.pdf (accessed July 2017). 